Hidalgo, H. G., Alfaro, E. J., \& Pérez-Briceño, P. M. (2021). Cambios climáticos proyectados de modelos CMIP5 en La Cruz, Guanacaste, Costa Rica. Revista de Biología Tropical, 69(Suppl. 2), S60-S73. https://doi.org/10.15517/ rbt.v69iS2.48307

\title{
Cambios climáticos proyectados de modelos CMIP5 en La Cruz, Guanacaste, Costa Rica
}

Hugo G. Hidalgo ${ }^{1,2}$; (D) https://orcid.org/0000-0003-4638-0742

Eric J. Alfaro ${ }^{1,2,3}$; (D) https://orcid.org/0000-0001-9278-5017

Paula M. Pérez-Briceño ${ }^{1,4,5}$; (D) https://orcid.org/0000-0002-7217-8495

1. Centro de Investigaciones Geofísicas, Universidad de Costa Rica, San José, Costa Rica; hugo.hidalgo@ucr.ac.cr, erick.alfaro@ucr.ac.cr, paula.perez@ucr.ac.cr

2. Escuela de Física, Universidad de Costa Rica, San José, Costa Rica.

3. Centro de Investigación en Ciencias del Mar y Limnología, Universidad de Costa Rica, San José, Costa Rica.

4. Escuela de Geografía, Universidad de Costa Rica, San José, Costa Rica.

5. Ingeniería Hidrológica, Sede Regional Chorotega, Universidad Nacional de Costa Rica, Guanacaste, Costa Rica.

Recibido 30-I-2021. Corregido 09-IV-2021. Aceptado 27-V-2021.

\section{ABSTRACT \\ Projected Climate Changes of CMIP5 models in La Cruz, Guanacaste, Costa Rica}

Introduction: La Cruz is located in northwestern Costa Rica, and it is characterized by a dry tropical climate with a well-defined dry season. This municipality has reported many impacts of hydrometeorological events during the last 45 years, mostly associated with wet events such as floods that affected roads, housing, and crops; as well as some droughts that have impacted the population and their livelihoods.

Objective: The objective of this article is to provide information regarding possible future climate changes during the $21^{\text {st }}$ century associated with a pessimistic emission scenario in the municipality of La Cruz in Guanacaste, Costa Rica.

Methods: We used DesInventar database to identify the hydrometeorological impacts in La Cruz and classified them by month. Downscaled simulations from the Climate Model Intercomparison Project 5 (CMIP5) were used to simulate past and future changes (1979-2099) in temperature and precipitation. In addition, a hydrological model was used to calculate runoff projections from meteorological data.

Results: Floods, and housing, roads and agricultural damages are the most recurrent impacts. In addition, some human deaths are reported during this time. Results show that a drier and warmer future will be characteristic of the climate in La Cruz in the $21^{\text {st }}$ century, especially after 2040. Runoff is also expected to be reduced significantly following the meteorological trends.

Conclusions: It is expected that higher aridity will be present in the future, affecting human and environmental systems.

Key words: climate change; hydrometeorological impacts; temperature; precipitation; Central American Dry Corridor; La Cruz; Costa Rica.

El municipio La Cruz en Costa Rica está ubicado en la vertiente del Pacífico, al noroeste del país en la frontera entre Costa Rica y Nicaragua. Este municipio y sus principales comunidades se muestran en la Fig. 1. La Cruz limita al norte con el departamento Rivas, Nicaragua; al sur con el cantón Liberia; al oeste con el Océano Pacífico; y al este con 


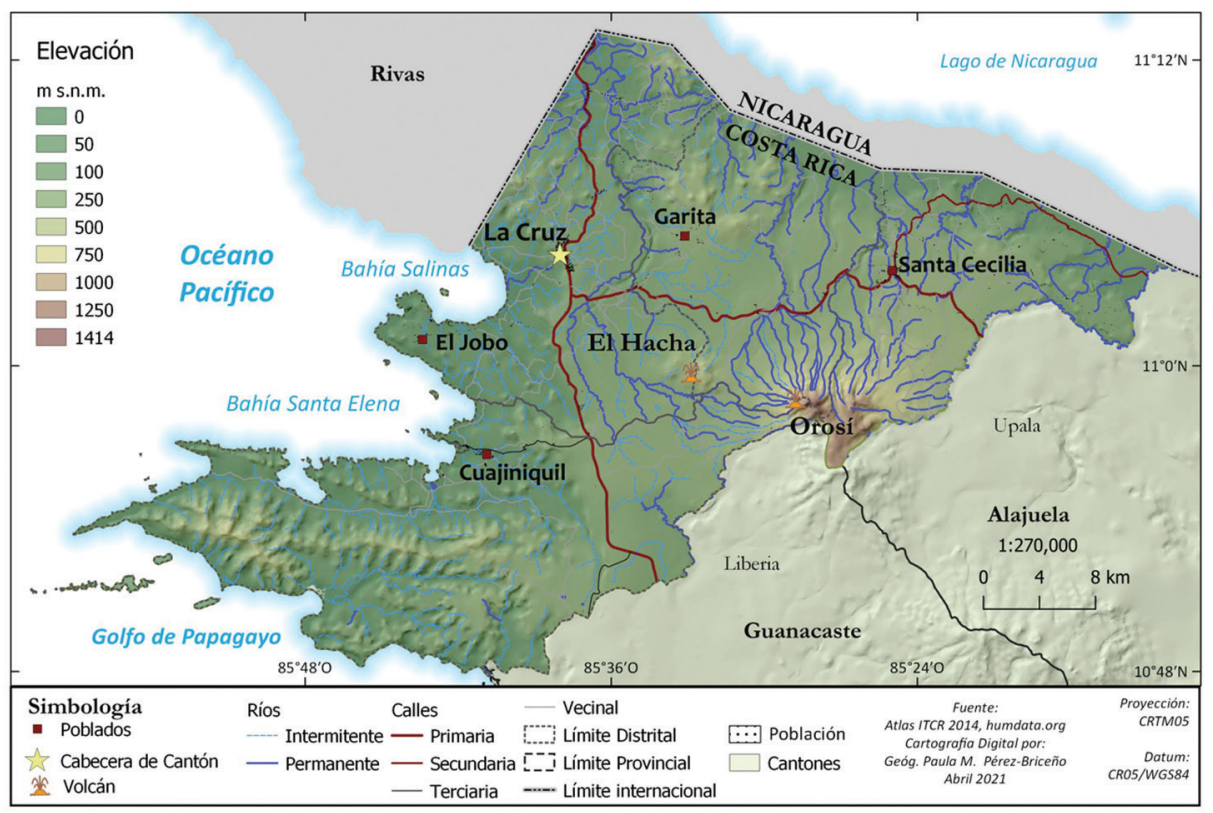

Fig. 1. Ubicación de las principales comunidades de la municipalidad La Cruz, Guanacaste, Costa Rica.

Fig. 1. Location of the main communities in the municipality of La Cruz, Guanacaste, Costa Rica.

el cantón Upala de la provincia Alajuela, los últimos tres pertenecientes a Costa Rica. A pesar de representar una limitada porción del corredor fronterizo (1 $389.87 \mathrm{~km}^{2}$, equivalentes a un $7.86 \%$ del área total del corredor fronterizo), se considera a La Cruz como un territorio altamente representativo de las dinámicas socioambientales, ya que posee zonas costeras y de altura con condiciones similares a las encontradas en el resto de dicho territorio fronterizo (Bautista-Solís et al., datos sin publicar). La amplitud máxima en La Cruz es de $81 \mathrm{~km}$, en dirección noreste a suroeste, lo cual facilita el traslado desde la zona costera a la zona más oriental donde predominan comunidades agropecuarias y forestales (Bautista-Solís et al., datos sin publicar). El territorio La Cruz comprende desde unos $150 \mathrm{~m}$ al noroeste del Hito 14, frontera con la República de Nicaragua, donde la línea imaginaria intercepta el río Las Haciendas hasta punta Santa Elena, en la península del mismo nombre. Las elevaciones son fluctuantes, pero no sobrepasan los 350 metros sobre el nivel medio del mar (m s.n.m.), a excepción del volcán Orosí que alcanza los
1400 m s.n.m. Algunos centros urbanos de los distritos del cantón son: Ciudad La Cruz 255 m s.n.m., Santa Cecilia 337 m s.n.m., La Garita 300 m s.n.m. y Cuajiniquil 10 m s.n.m. (Bautista-Solís et al., datos sin publicar).

Ruíz, Pérez-Briceño, Hidalgo y Alfaro (2019), así como Alfaro et al. (2012), describen y resumen los principales aspectos climáticos de esta región. De acuerdo con la clasificación del Instituto Meteorológico Nacional de Costa Rica [IMN] (http://www.imn.ac.cr) su régimen climático corresponde a la región del Pacífico Norte o seco, ya que presenta un período de disminución de lluvias bastante marcado entre los meses de diciembre y marzo, seguido por dos periodos de máxima precipitación: el primero en mayo-junio y el segundo, mayor que el primero, en agosto- setiembre-octubre. Dichos máximos están separados por un periodo de disminución de la precipitación, normalmente observado en julio, llamado "veranillo" (también conocido como veranillo de San Juan o canícula, Alfaro \& Hidalgo, 2017). Los meses de abril y noviembre se consideran periodos de transición entre las temporadas secas y lluviosas 
y viceversa. Según Alfaro et al. (2012), en esta región del Pacífico de Costa Rica, la magnitud del viento es normalmente más fuerte durante el invierno y primavera boreal (diciembre a mayo), con dirección predominante del este (vientos alisios) y disminuye durante el verano y otoño boreal, entre junio y noviembre.

Ruíz et al. (2019), muestran que las temperaturas superficiales del aire mínimas registradas en la estación de Santa Rosa, La Cruz, son en promedio de $26.5^{\circ} \mathrm{C}$. Estas presentan un máximo durante marzo-mayo, previo al inicio de la temporada lluviosa, y descienden durante el invierno boreal, siendo abril el mes de la máxima temperatura media y octubre el menor. Las temperaturas máximas menores ocurren durante septiembre-octubre, cuando se presenta la mayor cantidad de precipitación del ciclo anual. Las zonas costeras de La Cruz se encuentran en una zona donde ocurre un afloramiento costero (diciembre-abril), cuando los vientos alisios son más fuertes sobre la región y se presenta la época seca sobre la vertiente del Pacífico en América Central (Alfaro \& Cortés, 2012; Alfaro \& Cortés, 2021). La temperatura superficial del mar (TSM) media es de aproximadamente $25^{\circ} \mathrm{C}$. Según Alfaro y Cortés (2021), las temperaturas del mar más frías se observan en diciembre-abril, por debajo de $21^{\circ} \mathrm{C}$ en febrero y con un mínimo secundario en julio, lo que coincide con los máximos de la Corriente en Chorro de Bajo nivel del Caribe descrita por Amador (2008). Hay dos máximo de la TSM en mayo-junio y agosto-octubre con temperaturas superiores a $27{ }^{\circ} \mathrm{C}$ (superiores a $28^{\circ} \mathrm{C}$ en septiembre-octubre).

La interacción de factores como la temperatura oceánica, la intensidad de los vientos alisios, la intensidad de la corriente de choro del Caribe, la migración de la Zona de Inter-Convergencia Inter-Tropical (ZCIT), El Niño-Oscilación del Sur (ENOS), la Oscilación Decenal del Pacífico (PDO), entre otros, explican los mecanismos de la variabilidad climática de esta región de Guanacaste, Costa Rica (Bautista-Solís et al., datos sin publicar; Maldonado, Alfaro, \& Hidalgo, 2018).
La Cruz pertenece al Corredor Seco Centroamericano (CSC), una subregión del istmo relativamente más árida que el resto del territorio y que se extiende a lo largo del litoral pacífico desde el oeste de Guatemala hasta el norte de Costa Rica. En muchos estudios, el Arco Seco en Panamá también se considera parte del CSC. El CSC es una región definida de manera imprecisa que incluye características climáticas que favorecen el desarrollo de ecosistemas de bosque seco, con frecuentes períodos relativamente secos durante la temporada de lluvias (Hidalgo, Alfaro, Amador, \& Bastidas, 2019). El término corredor seco no solo es representativo de los efectos de los fenómenos climáticos, sino también refleja la ecología de esta región. El CSC es un área principalmente rural caracterizada por una marcada estacionalidad de las precipitaciones, vulnerabilidad al cambio climático, rica biodiversidad, pobreza arraigada, inseguridad alimentaria y emigración (Gotlieb, Pérez-Briceño, Hidalgo, \& Alfaro, 2019; Quesada-Hernández, Calvo-Solano, Hidalgo, Pérez-Briceño, \& Alfaro, 2019). Según Bautista-Solís et al. (datos sin publicar), La Cruz es particularmente vulnerable a la variabilidad climática. Por eso se requiere un arreglo institucional y tecnológico especial para la planeación del desarrollo regional (Bautista-Solís et al., datos sin publicar). En el 2017 y 2018, el Índice de Desarrollo Social y el Índice de Desarrollo Humano de La Cruz lo ubicaron en el cuartil más bajo de la comparación relativa de los municipios de Costa Rica (Mideplan, n.d.; PNUD, 2020). Estudios previos que delimitaron el corredor seco de Costa Rica, indican que el período de recurrencia de sequías prolongadas (multianuales) para la región Pacífico Central (incluye la provincia Guanacaste) varía entre 7.3 (Retana et al., 2012) a 10 años (Hidalgo et al., 2019), siendo esta región la que presenta los eventos más frecuentes, extensos y severos del país (Retana et al., 2012).

Según Hidalgo y Alfaro (2017), La Cruz contiene parte del Área de Conservación Guanacaste (ACG). Esta integra cuatro de los cinco ecosistemas principales del trópico: marino/ costero, bosque seco, bosque nuboso y bosque 
lluvioso y representa el único transecto conservado de este tipo en el neotrópico. En el ACG existen más especies terrestres que todas las que existen en el norte de México, Estados Unidos y Canadá juntos (ACG, 2012). Este ha sido uno de los argumentos fundamentales por lo que el Centro de Patrimonio Mundial de la UNESCO, declaró en 1999, al ACG Silvestre como Sitio Patrimonio de la Humanidad (ACG, 2012). El cambio climático podría traer impactos significativos en la sostenibilidad de las especies marinas y terrestres.

El objetivo de este trabajo es generar información acerca de los impactos hidrometeorológicos que han afectado a La Cruz en los últimos 45 años, así como determinar los posibles cambios climáticos durante el siglo XXI asociados con un escenario de emisiones pesimista en el cantón La Cruz. La estructura de este estudio es la siguiente: se continúa con la sección de materiales y métodos; luego los resultados son estructurados en una sección sobre los impactos producidos por eventos hidrometeorológicos, seguida por la modelación de los escenarios de cambio climático; finalmente, la discusión incluye un análisis de las implicaciones del incremento en la aridez sugerido por los escenarios de cambio climático.

\section{MATERIALES Y MÉTODOS}

Los impactos de eventos hidrometeorológicos más recurrentes registrados en La Cruz se recopilaron desde 1973 al 2018 (45 años), como línea base de las condiciones que podría enfrentar el cantón ante posibles cambios que se produzcan en el clima, de acuerdo con la base de datos DesInventar, accedida en 2020 (DesInventar, n.d.). Cabe resaltar que esta base de datos inicialmente responde a los reportes de periódicos nacionales principalmente y en años más recientes registra los datos oficiales por parte de las autoridades (Pérez-Briceño, Alfaro, Hidalgo, \& Jiménez, 2016; QuesadaRomán, Villalobos-Portilla, \& Campos-Durán, 2020). También es importante mencionar que los datos reportan fallecidos a causa de los eventos hidrometeorológicos, sin embargo, al no contar con el dato exacto, esta variable no se incluyó en el análisis. Estos datos se analizan en forma descriptiva.

Para el caso de los cambios climáticos futuros, se seleccionaron 14 simulaciones de modelos climáticos globales de circulación general, estas generan proyecciones climáticas mensuales de precipitación y temperatura del grupo llamado (CMIP5), AR5, del Panel Intergubernamental de Cambio Climático (IPCC en inglés). A pesar que recientemente se puso a disposición el los repositorios de datos las simulaciones de la generación CMIP6, aún no se dispone de la evaluación de estos modelos para América Central y el proceso de cambio de escala necesario. La selección de los modelos se basó en una recopilación de aquellos que mejor reproducen el clima histórico en América Central de acuerdo con Hidalgo y Alfaro (2015). Las diferencias y características de estos modelos se pueden ver en detalle en la referencia anterior.

Las simulaciones corresponden al camino representativo de concentración o "representative concentration pathway" referenciado como RCP8.5. Se seleccionó este escenario ya que al ser el más agresivo, es el que generará mayores impactos en el futuro. Los diferentes RCPs corresponden a los forzamientos de los gases de efecto invernadero (GEI) equivalentes en watts por metro cuadrado al final del siglo XXI. En otras palabras, el RCP8.5 corresponde a 8.5 watts $/ \mathrm{m}^{2}$ o $\sim 1370 \mathrm{ppm} \mathrm{CO}_{2}$ equivalente a final de siglo por efectos de GEI y por consiguiente, y se considera un escenario agresivo o pesimista con respecto a las emisiones de gases de efecto invernadero y en el que los efectos antrópicos son mayores. Los números que aparecen luego del nombre del modelo en los nombres de los archivos son el número de simulación (r), que corresponde a diferentes versiones del modelo con la misma física, pero estados iniciales (realizaciones), método de inicialización (i) y diferentes físicas (p). La lista de modelos se muestra en la Tabla 1.

Estos modelos globales tienen una resolución muy gruesa (del orden de $250 \mathrm{~km}$ x 250 $\mathrm{km}$ o más), así que necesitan un procedimiento 
TABLA 1

Listado de modelos y simulaciones usadas para proyectar los escenarios climáticos de la municipalidad La Cruz, Guanacaste, Costa Rica

TABLE 1

List of models and runs used for projecting the climate change scenarios for the municipality of La Cruz, Guanacaste, Costa Rica

\begin{tabular}{cl}
\hline Número & \multicolumn{1}{c}{ Nombre del modelo y simulación } \\
\hline 1 & ccsm4_r1i1p1 \\
2 & ccsm4_r2i1p1 \\
3 & cesm1_cam5_r1i1p1 \\
4 & cesm1_cam5_r2i1p1 \\
5 & cmec_cmsr1i1p1 \\
6 & ec_earth_r2i1p1 \\
7 & giss-e2_r_r1i1p1 \\
8 & miroc5_r1i1p1 \\
9 & miroc5_r3i1p1 \\
10 & mpi_esm_lr_r1i1p1 \\
11 & mpi_esm_lr_r2i1p1 \\
12 & mpi_esm_lr_r3i1p1 \\
13 & ccsm4_r3i1p1 \\
14 & cesm1_cam5_r3i1p1 \\
\hline
\end{tabular}

denominado "cambio de escala" (Amador \& Alfaro, 2009), para obtener los datos a una resolución más adecuada para el análisis a escala municipal (en este caso $5 \mathrm{~km}$ x $5 \mathrm{~km}$ ). Esto se hizo de forma estadística de acuerdo con Hidalgo, Alfaro y Quesada-Montano (2017). Se calcularon los cambios en precipitación y temperatura, con respecto al clima histórico (1979-1999 como periodo base). Por medio de un modelo de tiempo atmosférico, se transformaron los datos de precipitación y temperatura de resolución temporal mensual a diaria de acuerdo con el procedimiento explicado en Alfaro-Córdoba, Hidalgo y Alfaro (2020); para obtener los datos que se usaron en un modelo hidrológico y obtener la escorrentía total (superficial más flujo base). Este modelo se simuló 1000 veces con los datos meteorológicos diarios, junto con parámetros variados al azar siguiendo los rangos encontrados en Hidalgo, Amador, Alfaro y Quesada (2013). El modelo hidrológico utilizado es el modelo de macroescala conocido como Capacidad de Infiltración Variable (VIC; Liang,
Lettenmaier, Wood, \& Burges, 1994) y se generó la proyección de la escorrentía futura promedio en la región de 1979 a 2099. El modelo VIC ha demostrado simular con gran destreza parámetros hidrológicos en distintas regiones del mundo, incluyendo América Central (Hidalgo et al., 2009; Hidaldo et al., 2013; Lin et al., 2019).

\section{RESULTADOS}

\section{Impactos de eventos hidrometeoroló- gicos en La Cruz, Guanacaste, Costa Rica:} En este apartado, se caracterizan los impactos hidrometeorológicos registrados en La Cruz. La Fig. 2 presenta la distribución de los impactos durante el año, donde se aprecia que la mayor cantidad de impactos se producen durante la época lluviosa. Las inundaciones y las sequías son los impactos más recurrentes en el periodo analizado de 45 años (1973-2018). Estos eventos han generado afectaciones principalmente por daños a viviendas e infraestructura vial. La Fig. 2 muestra una distribución bimodal con dos máximos, uno en julio y otro en septiembre y octubre mayor que el primero, mientras que en agosto se muestra un descenso de los impactos reportados. Esto está de acuerdo con la distribución de las precipitaciones observadas en el municipio (Rodríguez, Alfaro, \& Cortés, 2021).

Los impactos se pueden categorizar en húmedos, que tienen un impacto en la escala temporal más cortas; y secos, con una escala temporal que puede durar varios meses. Así que, aunque se reportan una menor cantidad de eventos secos, la recuperación posterior al impacto puede ser mayor en tiempo y dinero. Bautista-Solís et al. (datos sin publicar) agregan que el cantón La Cruz enfrenta una alta exposición a eventos hidrometeorológicos extremos como las sequías y las inundaciones y además es un cantón vulnerable en términos de desarrollo humano (Díaz, Mora, \& Madriz, 2019). Durante el huracán Otto en La Cruz llovió más de $250 \mathrm{~mm}$ en menos de 6 horas (CNE, 2017). En adición, durante la tormenta tropical Nate (2017), por ejemplo, se registraron 


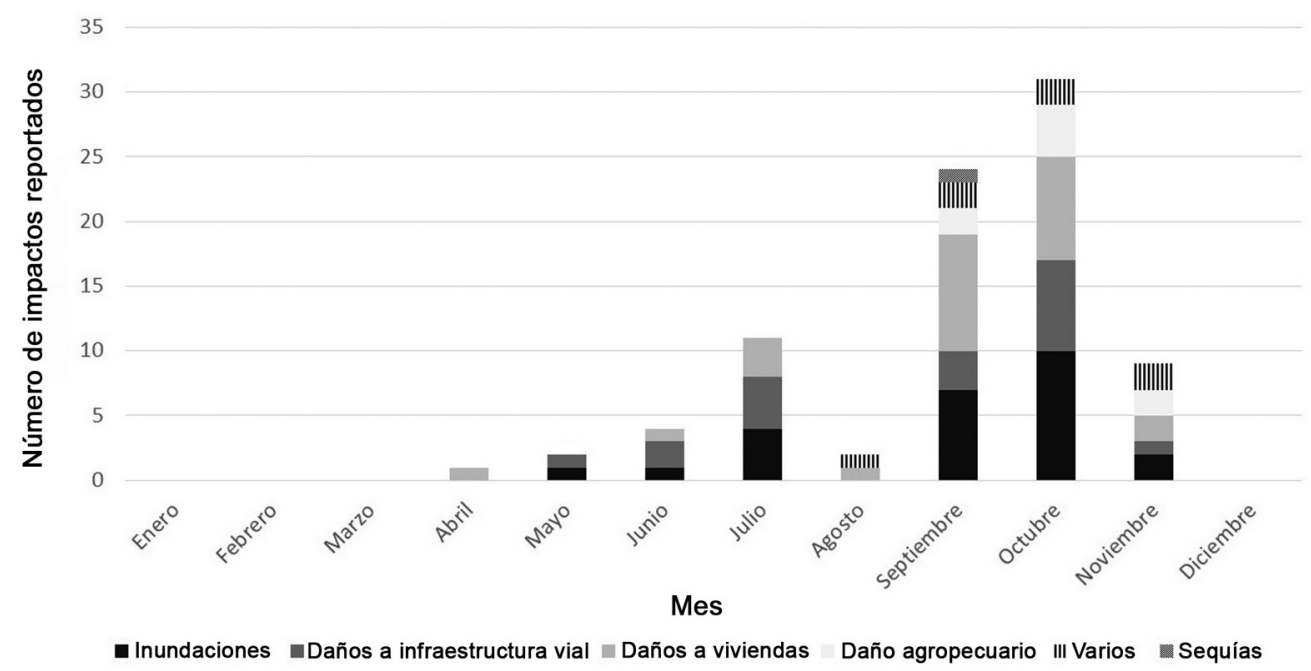

Fig. 2. Distribución mensual de los impactos por eventos hidrometeorológicos en el cantón La Cruz, 1973-2018 (datos obtenidos de la base Desinventar, 2020). La categoría varios se refiere a impactos como: daños en alcantarillas, daños en el servicio eléctrico o comunicación, problemas para navegar o volar, caída de árboles, reporte de heridos o afectados.

Fig. 2. Annual cycle of the hydrometeorological event impacts in the municipality La Cruz, 1973-2018 (Data from Desinventar, 2020). The varios (others) category refers to impacts such as: damages to culverts, damages in electrical and communication networks, problems for sea and air navigation, fallen trees, reports of affected or wounded people.

precipitaciones superiores a los $400 \mathrm{~mm}$ en $\mathrm{La}$ Cruz que saturaron el suelo y provocaron inundaciones y que afectaron además a 76 cantones del país (Alfaro, Hidalgo, Maldonado, PérezBriceño, \& Mora, 2018).

\section{"Escenarios" de Cambio Climático}

Los cambios proyectados en la precipitación en los próximos 40 años (2040-2060) no son muy grandes (Fig. 3), aún para este escenario pesimista. Por el contrario, los cambios proyectados en esta variable al final de siglo (2079-2099) evidencian disminuciones del orden de hasta $-10 \%$ en la parte suroeste del cantón La Cruz y, de alrededor de $-5 \%$ en la parte más oriental del cantón, caracterizada por ser una zona más montañosa y con mayor influencia en el clima de la vertiente del Caribe (Fig. 1). Lo que coincide en términos generales por lo reportado para la región por Hidalgo et al. (2017), Hidalgo y Alfaro (2017), Imbach et al. (2018) así como Moreno, Hidalgo y Alfaro (2019). Alfaro-Córdoba et al. (2020) encontraron una tendencia negativa para el periodo
1970-1999 en la estación de Liberia en Guanacaste, adicionalmente, Hannah et al. (2017), observaron una tendencia histórica negativa para esta región al utilizar las bases de datos del CPC Merged Analysis of Precipitation (CMAP; Xie \& Arkin, 1997) y el reanálisis del NCEP/ NCAR, para el periodo 1982-2005 (Kalnay et al., 1996).

En el caso de la temperatura (Fig. 4), tanto a mediados de siglo (2040-2060) como a finales (2079-2099), se proyectan incrementos de aproximadamente $2{ }^{\circ} \mathrm{C}$ a $5{ }^{\circ} \mathrm{C}$ para ambas épocas respectivamente, en la mayoría de $\mathrm{La}$ Cruz y ligeramente menores en las partes altas del cantón. Estos resultados coinciden con lo obtenido para la región en otros estudios (e.g. Alfaro et al., 2012; Alfaro-Córdoba et al., 2020; Hidalgo \& Alfaro, 2017; Hidalgo et al., 2017; Imbach et al., 2012; Imbach et al., 2018; Moreno et al., 2019), quienes observaron una tendencia histórica positiva para la temperatura superficial del aire (1970-1999) y del mar (1854-2011) en esta región. Los avances en el conocimiento de la variabilidad de la temperatura en La Cruz son importantes para los asentamientos costeros de 
A Mediana de precipitación anual

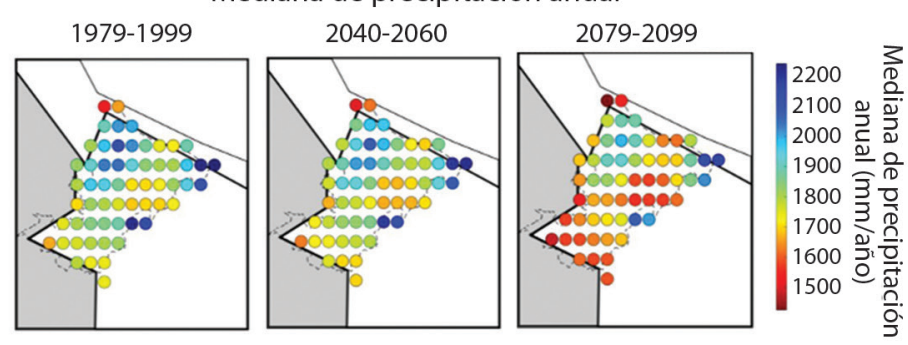

B

Cambio con respecto a 1979-1999
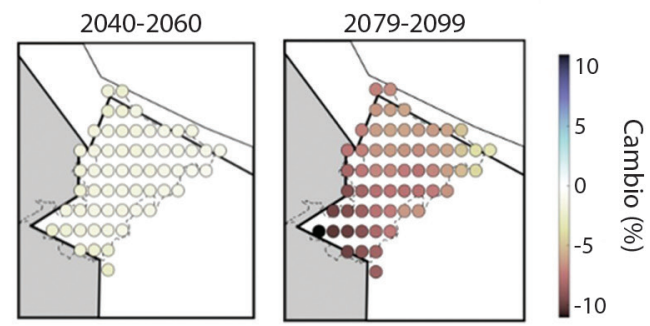

Fig. 3. (a) Promedios anuales (mm/año) de precipitación bajo un escenario pesimista de concentraciones en diferentes períodos calculados como la mediana de los ensambles de los modelos CMIP5 para el cantón de La Cruz, Guanacaste. (b) Cambios relativos al período histórico 1979-1999 (b). El Océano Pacífico está sombreado en gris.

Fig. 3. (a) Precipitation annual means ( $\mathrm{mm} /$ year) for different periods under a pessimistic scenario computed as the ensemble median for CMIP5 models shown in Table 1 at La Cruz municipality, Guanacaste. (b) Changes relative to the historical period 1979-1999. The Pacific Ocean is shaded in grey.

pescadores, ya que las anomalías positivas y negativas de la temperatura del mar observadas están relacionadas con las capturas comerciales de peces en el Pacífico norte costarricense (Moreno \& Alfaro, 2018; Moreno, Moya, \& Alfaro, 2017) y la pesca es uno de los sectores socioeconómicos de mayor importancia en esta región. Según Alfaro \& Lizano (2001), El NiñoOscilación del Sur (ENOS) domina la señal interanual de la variabilidad en la TSM en la región.

Los valores agregados para todo el cantón La Cruz (Fig. 5) confirman lo sugerido con anterioridad, observándose que la reducción en precipitación empieza a registrarse alrededor del año 2040; mientras que la temperatura tiene una tendencia monotónica para toda la simulación. En esta figura también se evidencia en la dispersión de los gráficos de cajas, hay menor incertidumbre entre los modelos en las proyecciones de temperatura que de precipitación.
Los modelos de Circulación General (GCMs en inglés), han demostrado tener mucho menor destreza en reproducir patrones climáticos de precipitación comparados con los de temperatura (Hidalgo \& Alfaro, 2015). En general, los cambios sugieren condiciones más secas y cálidas en el cantón a finales de siglo.

En cuanto a la escorrentía total modelada (Fig. 6), se notan reducciones importantes en este parámetro a partir de 2040, precisamente como una combinación de las reducciones en precipitación y aumentos en temperatura en ese período. Hidalgo et al. (2013) encontraron una reducción de la escorrentía superficial para la proyección del siglo XXI en la estación de Liberia en Guanacaste. Lo anterior coincide con lo encontrado por Hidalgo \& Alfaro (2017), Moreno et al. (2019), Alfaro-Córdoba et al. (2020), para el municipio La Cruz, como parte del CSC. 
A Mediana de temperatura anual
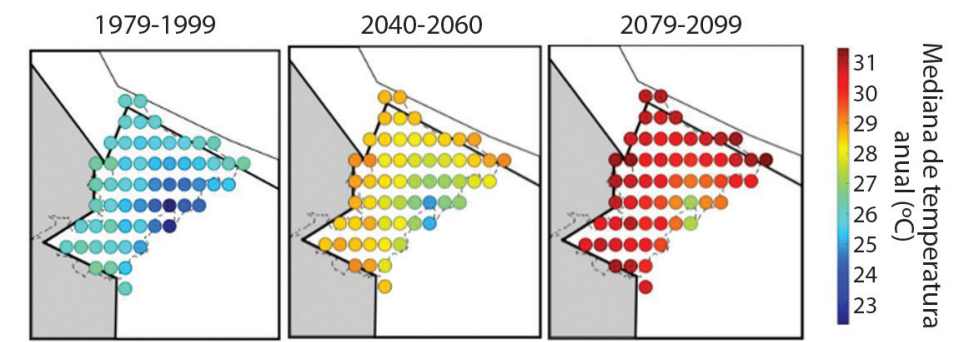

B
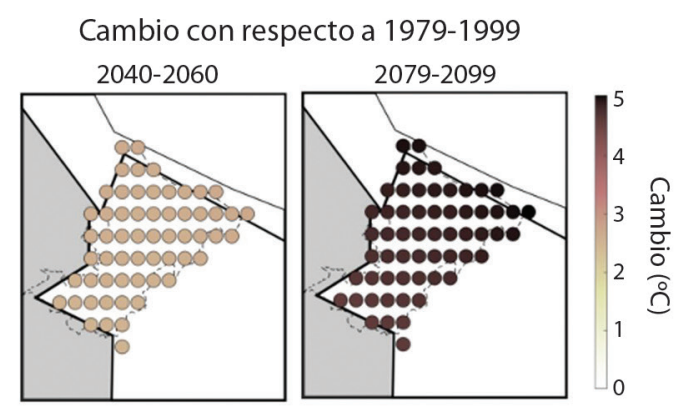

Fig. 4. (a) Promedios anuales $\left({ }^{\circ} \mathrm{C}\right)$ de temperatura bajo un escenario pesimista de concentraciones en diferentes períodos calculados como la mediana de los ensambles de los modelos mostrados CMIP5 para el cantón La Cruz, Guanacaste. (b) Cambios relativos al período histórico 1979-1999. El Océano Pacífico está sombreado con gris.

Fig. 4. (a) Temperature annual means $\left({ }^{\circ} \mathrm{C}\right)$ for different periods under a pessimistic scenario computed as the ensemble median for CMIP5 models shown in Table 1 at La Cruz municipality, Guanacaste. (b) Changes relative to the historical period 1979-1999. The Pacific Ocean is shaded in grey.

\section{DISCUSIÓN}

Las tendencias del clima en La Cruz sugieren un futuro más seco y caliente a finales del siglo XXI. Los cambios en precipitación al final de siglo (2079-2099) evidencian cambios del orden de hasta $-10 \%$ en la parte suroeste del cantón, y de alrededor de - $5 \%$ en la parte más este del cantón, caracterizada por ser una zona más montañosa. En el caso de la temperatura, tanto a mediados de siglo (20402060) como a finales (2079-2099), se proyectan calentamientos de aproximadamente $2{ }^{\circ} \mathrm{C}$ a $5{ }^{\circ} \mathrm{C}$ para cada época, respectivamente, y para la mayoría del área, aunque ligeramente menos en las partes altas del cantón. Los resultados de este estudio son consistentes con otras áreas estudiadas con una escala de sus modelos similar, con precipitaciones menores a $2000 \mathrm{~mm}$ y con un ecosistema contrastante como lo es el páramo del Parque Nacional Chirripó en Costa Rica (Veas-Ayala, Quesada-Román, Hidalgo \& Alfaro, 2018).

Según Moreno et al. (2019), este aumento en la temperatura acompañado de una disminución en la precipitación también afectará los servicios ecosistémicos brindados por el bosque tropical seco. Lo anterior puede traer consigo repercusiones importantes en el ámbito ecológico y de disponibilidad de agua futura. Además, debido a la presencia de bosques secos en la zona y la alta productividad de biomasa, aunado al aumento de la temperatura pueden crear una alta vulnerabilidad en la zona a la ocurrencia de incendios forestales. Estas condiciones han sido reportadas en asocio con sus características geomorfológicas muy similares a las del cantón La Cruz en la ACG por Vargas-Sanabria y Quesada-Román (2018). 

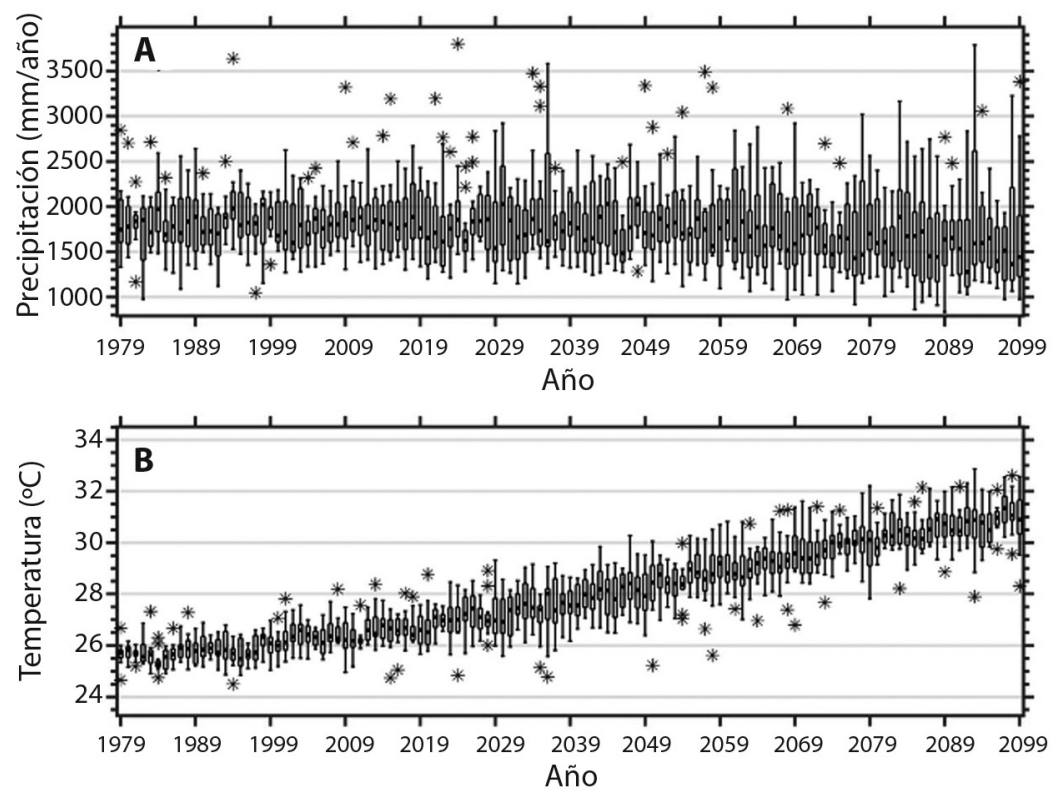

Fig. 5. Valores anuales proyectados bajo un escenario pesimista de concentraciones en la (a) precipitación y (b) temperatura de 1979 a 2099 promediado para todo el cantón de La Cruz. Las cajas en el diagrama muestran la variabilidad de todos los modelos CMIP5, $\mathrm{N}=14$ modelos y simulaciones climáticas de la Tabla 1.

Fig. 5. Annual values of (a) precipitation and (b) temperature under a pessimistic scenario from 1979 to 2099 averaged for all the La Cruz municipality. The boxes of the diagram show the variability of all CMIP5 models in Table $1(\mathrm{~N}=14)$.

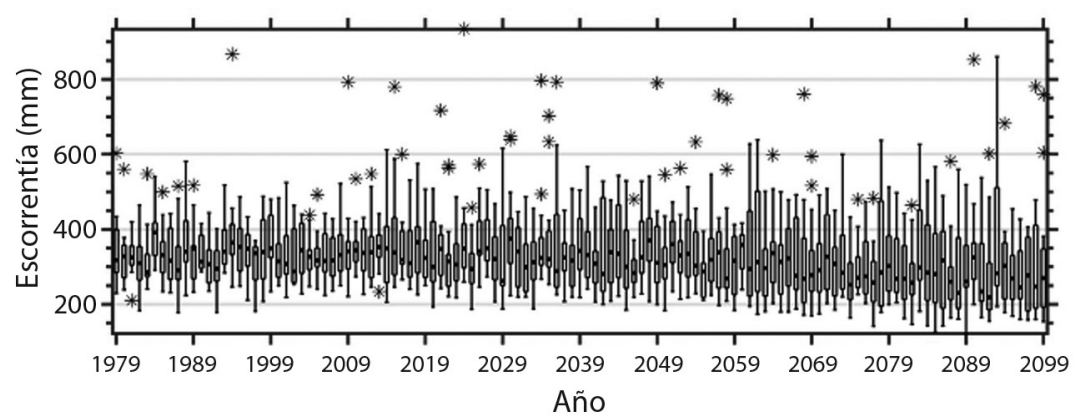

Fig. 6. Valores anuales de mediana para la escorrentía total (superficie + flujo base) simulados con el modelo de Capacidad de Infiltración Variable (VIC) en el municipio La Cruz, Guanacaste, Costa Rica. Para cada una de las 14 simulaciones de la Tabla 1, se escogió la mediana de 1000 simulaciones del modelo hidrológico VIC.

Fig. 6. Median annual total runoff values (surface + baseflow) simulated by the Variable Infiltration Capacity (VIC) model for the municipality La Cruz, Guanacaste, Costa Rica. For each of the 14 simulations from Table 1, the median of 1000 simulations of VIC hydrological model were chosen.

Moreno et al. (2019) agregan que lo anterior puede agravarse porque el grado de incentivos para la conservación del bosque en terrenos privados, derivados de las políticas ambientales no son suficientes para asegurar que el bosque existente en Guanacaste pueda estar protegido en contra de los efectos potencialmente negativos de cambios socioeconómicos futuros.

La combinación de ambos efectos produce una respuesta amplificada en las proyecciones de escorrentía de 1979 a 2099 en la segunda mitad del siglo XXI, y se notan reducciones 
importantes en este parámetro a partir de alrededor 2040. Precisamente, esta tendencia es una combinación de las reducciones en precipitación y aumentos en temperatura en ese periodo. Esto por cuanto la disminución en la precipitación y el aumento en la evapotranspiración, debido al aumento en la temperatura, traerá consigo suelos más secos y menos agua disponible para producir escorrentía superficial, afectando también la infiltración y recarga de los sistemas acuíferos. Las tendencias positivas de temperatura pueden aumentar la aridez y disminuir la escorrentía a través de la intensificación del ciclo hidrológico regional (Vargas-Sanabria \& Quesada-Román, 2018).

El análisis sugiere que, si las condiciones del clima se vuelven más áridas, las medidas de mitigación y adaptación desde la gestión del riesgo deben estar orientadas en maximizar la gestión del recurso hídrico, tanto en la estación seca como lluviosa, ya que los impactos hidrometeorológicos que más han afectado al cantón están relacionados con la precipitación, ya sea por un evento extremo húmedo o al contrario por un periodo de sequía. Cabe destacar que la Figura 1 muestra la distribución de la población en el cantón, donde se observa que se sitúa en las partes bajas y de llanura, muy cerca de cauces fluviales, condición que en una crecida del río afectaría de manera directa al medio de vida de las comunidades (CamposDurán \& Quesada-Román, 2017; Office for the Coordination of Humanitarian Affairs, 2020). Estas condiciones extremas que podrían afectar a La Cruz en un evento extraordinario de precipitación asociado con un ciclón tropical fueron explicados a detalle para el cantón adyacente, Upala (Quesada-Román, Fallas-López, Hernández-Espinoza, Stoffel, \& BallesterosCánovas, 2019; Quesada-Román \& VillalobosChacón, 2020). A pesar de que Upala está en la vertiente Caribe, La Cruz tiene condiciones geomorfológicas similares y podrían darse eventos intensos asociados con flujos de lodo o bien inundaciones dada la rápida transición entre la mencionada zona montañosa y las llanuras donde se ubica la población.
La Cruz aún mantiene condiciones de ruralidad, grandes parcelas y población dispersa, excepto en la cabecera de cantón (Fig. 1), situación que no lo exime de gestionar su territorio. Tal y cómo lo señalan Quesada-Román et al. (2020), la combinación de ser un lugar altamente expuesto a amenazas hidrometeorológicas, la variabilidad y cambio climático, y la falta de un eficiente ordenamiento territorial, podría significar pérdidas económicas para el cantón a corto y mediano plazo. En concordancia con Moreno et al. (2019), al analizar los resultados de los escenarios climáticos, se puede concluir que se presentará un efecto sobre los servicios ecosistémicos en el cantón estudiado, debido a la presencia de ecosistemas vulnerables al cambio climático.

La generación de escenarios de Cambio Climático en regiones de índice de desarrollo social bajo como el cantón La Cruz, aporta información para la toma de decisión, ya que según Bautista-Solís et al. (datos sin publicar), los sectores más vulnerables de la población tienen dificultades para orientar la toma de decisiones en adaptación para recursos estratégicos como el agua, suelo y bosques con información científico-técnica, por lo que es necesario gestionar la información para que sea accesible para toda la población. Además, históricamente, La Cruz se ha visto afectada por diversas amenazas hidrometeorológicas que han venido a impactar directamente a la población. Estas se expresan localmente como inundaciones que dañan tanto a viviendas como infraestructura vial; y también de manera considerable los efectos que tienen las sequías al ser un evento de una escala temporal más extendida. Por lo tanto, adaptarse a las variaciones y cambios climáticos es un paso fundamental para la resiliencia de las comunidades rurales y para facilitar los esfuerzos de desarrollo territorial rural de la mano con el ordenamiento territorial de la región. Las zonas transfronterizas como La Cruz poseen un contexto muy particular que influye en los alcances del desarrollo rural de América Central. Por un lado, se cuenta progresivamente con más información sobre la influencia de aspectos biofísicos y climáticos 
en la variabilidad y cambio climático y los medios de vida locales; por otro, hay una necesidad constante de adaptar políticas y opciones de adaptación a los intereses de tomadores de decisiones y población, políticas internacionales, efectos de la globalización y el imaginario del territorio (Bautista-Solís et al., datos sin publicar). Obsérvese que en toda aplicación de los modelos climáticos, así como del escenario escogido para la adaptación a escala municipal, se deben considerar los aspectos descritos por Nissan, Goddard, de Perez y Furlow (2019), y por Hidalgo (2021), para orientar la adecuada participación de los actores en el proceso de retroalimentación.

Declaración de ética: los autores declaran que todos están de acuerdo con esta publicación y que han hecho aportes que justifican su autoría; que no hay conflicto de interés de ningún tipo; y que han cumplido con todos los requisitos y procedimientos éticos y legales pertinentes. Todas las fuentes de financiamiento se detallan plena y claramente en la sección de agradecimientos. El respectivo documento legal firmado se encuentra en los archivos de la revista.

\section{AGRADECIMIENTOS}

Se agradece el apoyo de los siguientes proyectos inscritos en la Vicerrectoría de Investigación de la Universidad de Costa Rica: B9-454 (VI-Grupos), EC-497 (FEES-CONARE), C0-610 (Fondo de Estímulo), A4-906 (PESCTMA-CIGEFI), C0-404 (PNUD), C0-074, A1-715, B0-810 y A5-037. A la Escuela de Física de la UCR por darnos el tiempo de investigación para desarrollar este estudio. Al centro de investigación CIGEFI de la UCR por su apoyo logístico durante la recopilación y análisis de datos.

\section{RESUMEN}

Introducción: La Cruz está localizada en el noroeste de Costa Rica, y está caracterizada por una estación seca bien definida y un clima tropical seco. Este cantón en los últimos 45 años ha reportado impactos de eventos hidrometeorológicos, en particular los húmedos como las inundaciones que han afectado viviendas, calles y cultivos, pero también algunas sequías han perjudicado a la población y sus actividades.

Objetivo: El objetivo de este artículo es proveer información acerca de posibles cambios climáticos durante el siglo XXI asociados con un escenario pesimista de emisiones en el cantón de La Cruz en Guanacaste, Costa Rica.

Métodos: Se utilizó la base de datos DesInventar para identificar los impactos y agruparlos según su mes de ocurrencia para relacionarlo con el régimen de precipitación. Simulaciones cambiadas de escala del Proyecto de Intercomparación de Modelos Climáticos 5 (CMIP5) fueron estados para simular cambios pasados y futuros (19792099) en temperatura y precipitación. Adicionalmente, un modelo hidrológico fue usado para calcular las proyecciones de escorrentía usando los datos meteorológicos.

Resultados: Los resultados mostraron que un futuro más seco y caliente será característico del clima del siglo XXI, especialmente después del 2040. La escorrentía se espera que será reducida significativamente siguiendo las tendencias meteorológicas.

Conclusiones: Algunos de los impactos más recurrentes históricamente son las inundaciones, daños a viviendas, calles y cultivos, sin embargo, también se han registrado algunas muertes en la zona. Se espera que una aridez mayor estará presente en el futuro, afectando sistemas humanos y ambientales.

Palabras clave: Cambio Climático, impactos hidrometeorológicos, temperatura, precipitación, Corredor Seco Centroamericano, Municipio La Cruz, Costa Rica.

\section{REFERENCIAS}

ACG. (2012). ¿Qué es el Área de Conservación Guanacaste? Área de Conservación Guanacaste. https://www. acguanacaste.ac.cr/acg/que-es-el-acg

Alfaro, E. J., \& Cortés, J. (2012). Atmospheric forcing of cool subsurface water events in Bahía Culebra, Gulf of Papagayo, Costa Rica. Revista de Biología Tropical, 60(Supplement 2), S173-S186.

Alfaro, E. J., \& Cortés, J. (2021). Climate forcing of cool and warm subsurface water events in Bahía Salinas, Costa Rica. Revista de Biología Tropical, 69(Suppl. 2), S127-S141.

Alfaro, E. J., Cortés, J., Alvarado, J. J., Jiménez, C., León, A., Sánchez-Noguera, C., Nivia-Ruiz, J., \& Ruiz, E. (2012). Clima y temperatura superficial del mar en Bahía Culebra, Golfo de Papagayo, Costa Rica. Revista de Biología Tropical, 60(Supplement 2), S159-S171. 
Alfaro, E. J., \& Hidalgo, H. G. (2017). Propuesta metodológica para la predicción climática estacional del veranillo en la cuenca del río Tempisque, Costa Rica, América Central. Tópicos Meteorológicos y Oceanográficos, 16(1). 62-74.

Alfaro, E. J., Hidalgo, H. G., Maldonado, T., Pérez-Briceño, P.M., \& Mora, N.P. (2018). A tri-dimensional approach to climate sciences. Lessons from a Central American university. Caribbean Quarterly, 64(1), 26-56. https://doi.org/10.1080/00086495.2018.1435 333

Alfaro, E. J., \& Lizano, O. G. (2001). Algunas relaciones entre las zonas de surgencia del Pacífico Centroamericano y los Océanos Pacífico y Atlántico Tropical. Revista de Biología Tropical, 49(Supplement 2), S185-S193.

Alfaro-Córdoba, M., Hidalgo, H. G., \& Alfaro, E. J. (2020). Aridity trends in Central America: A spatial correlation analysis. Atmosphere, 11(4), 427. https://doi. org/10.3390/atmos 11040427

Amador, J. A. (2008). The intra-Americas seas low-level jet (IALLJ): Overview and future research. Annals of the New York Academy of Sciences, 1146(1), 153-188. https://doi.org/10.1196/annals.1446.012

Amador, J., \& Alfaro, E. J. (2009). Métodos de reducción de escala: Aplicaciones al clima, variabilidad y cambio climático. Revista Iberoamericana de Economía Ecológica, 11, 39-52.

Bautista-Solís, P., Hidalgo, H. G., Alfaro, E. J., Sáenz, F., Suárez, A., Pérez-Briceño, P. M., Moreno, M. L., Golcher, C., \& Ramírez, J. C. (Datos sin publicar). Metodología para el fortalecimiento a la resiliencia multisectorial a la variabilidad climática. El caso del proyecto "VarClim" en el cantón La Cruz, Guanacaste, Costa Rica.

Campos-Durán, D., \& Quesada-Román, A. (2017). Impacto de los eventos hidrometeorológicos en Costa Rica, periodo 2000-2015 / Impact of the hydrometeorological events in Costa Rica, periodo 2000-2015. Geo UERJ, 30, 440-65. https://doi.org/ 10.12957/ geouerj.2017.2611610.12957/geouerj.2017.26116.

CNE - Comisión Nacional de Prevención de Riesgos y Atención de Emergencias (2017). Plan general de la emergencia ante la situación provocada por el paso del huracán Otto por territorio costarricense. San José, Costa Rica. pp 25. Recuperado de https:// www.cne.go.cr/Documentos/planes-emergencia/ plan_emergencia_40027.pdf

DesInventar. (n.d.). DesInventar. United Nations Office for disaster risk reduction. https://www.desinventar. net/DesInventar/
Díaz, R., Mora, V., \& Madriz, G. (2019). El desenvolvimiento histórico de un asentamiento humano en el Corredor Seco Centroamericano (CSC): Cuajiniquil de La Cruz, provincia de Guanacaste, Costa Rica (1940-2018). In C. García \& C. Lértora (Eds.), Ciencias ambientales y participación ciudadana: Proyecto EcoEpisteme (pp. 161-212). Buenos Aires, Argentina: FEPAI.

Gotlieb, Y., Pérez-Briceño, P. M., Hidalgo, H. G., \& Alfaro, E. J. (2019). The Central American Dry Corridor: A Consensus Statement and its Background. "Yu'am" Revista Mesoamericana de Biodiversidad y Cambio Climático, 3(5), 42-51.

Hannah, L., Donatti, C., Harvey, C., Alfaro, E., Rodriguez, D., Bouroncle, C., Castellanos, E., Diaz, F., Fung, E., Hidalgo, H., Imbach, P., Laderach, P., Landrum, J., \& Solano, A. (2017). Regional modeling of climate change impacts on smallholder agriculture and ecosystems in Central America. Climatic Change, 141, 29-45. https://doi.org/10.1007/ s10584-016-1867-y

Hidalgo, H. G. (2021). Climate variability and change in Central America: What does it mean for water managers? Frontiers in Water, 2, 632739. https://doi. org/10.3389/frwa.2020.632739

Hidalgo, H. G. \& Alfaro, E. J. (2015). Skill of CMIP5 climate models in reproducing 20th century basic climate features in Central America. International Journal of Climatology, 35, 3397-3421. https://doi. org/10.1002/joc.4216

Hidalgo, H. G., \& Alfaro, E. J. (2017). Proyecciones de cambio climático en la parte terrestre del Área de Conservación Guanacaste (ACG). Documento técnico, CIGEFI-UCR. Recuperado de http://hdl.handle. net/10669/81741

Hidalgo, H. G., Alfaro, E. J., Amador, J. A., \& Bastidas, A. (2019). Precursors of quasi-decadal dry-spells in the Central America Dry Corridor. Climate Dynamics, 53(3-4), 1307-1322. https://doi.org/10.1007/ s00382-019-04638-y

Hidalgo, H. G., Alfaro, E. J., \& Quesada-Montano, B. (2017). Observed (1970-1999) climate variability in Central America using a high-resolution meteorological dataset with implication to climate change studies. Climatic Change, 141, 13-28. https://doi. org/10.1007/s10584-016-1786-y

Hidalgo, H. G., Amador, J. A., Alfaro, E. J., \& Quesada, B. (2013). Hydrological climate change projections for Central America. Journal of Hydrology, 495, 94-112.

Hidalgo, H. G., Das, T., Dettinger, M. D, Cayan, D. R., Pierce, D. W., Barnett, T. P., Bala, G., Mirin, A., Wood, A. W.; Bonfils, C., Santer, B. D., \& Nozawa, 
T. (2009). Detection and attribution of streamflow timing changes to climate change in the Western United States. Journal of Climate, 22, 3838-3855. https:// doi.org/10.1175/2009JCLI2470.1

Imbach, P., Chou, S. C., Lyra, A. et al. (2018). Future climate change scenarios in Central America at high spatial resolution. PLOS ONE, 13, e0193570. https:// doi.org/ 10.1371/journal.pone.0193570

Imbach, P., Molina, L., Locatelli, B., Roupsard, O., Mahe, G., Neilson, R., Corrales, L., Scholze, M., \& Ciais, P. (2012). Modeling potential equilibrium states of vegetation and terrestrial water cycle of Mesoamerica under climate change scenarios. Journal of Hydrometeorology, 13, 665-680. https://doi.org/10.1175/ JHM-D-11-023.1

Kalnay, E., Kanamitsu, M., Kistler, R., Collins, W., Deaven, D., Gandin, L., Iredell, M., Saha, S., White, G., Woollen, J., Zhu, Y., Leetmaa, A., \& Reynolds, R. (1996). The NCEP/NCAR 40-year reanalysis project. Bulletin of the American Meteorological Society, 77, 437-471. https://doi. org/10.1175/1520-0477(1996)077<0437:TNYRP $>2$. $0 . \mathrm{CO} ; 2$

Liang, X., Lettenmaier, D. P., Wood, E. F., \& Burges, S. J. (1994). A simple hydrologically based model of land surface water and energy fluxes for general circulation models. Journal of Geophysical Research, 99, 14415-14428.

Lin, P., Pan, M., Beck, H. E., Yang, Y., Yamazaki, D., Frasson, R., David, C. H., Durand, M., Pavelsky, T. M., Allen, G. H., Gleason, C. J., \& Wood, E. F. (2019). Global reconstruction of naturalized river flows at 2.94 Million Reaches. Water Resources Research, 55, 6499-6516. https://doi.org/10.1029/2019WR025287

Maldonado, T., Alfaro, E. J., \& Hidalgo H. G. (2018). Revision of the main drivers and variability of Central America Climate and seasonal forecast systems. Revista de Biología Tropical, 66(Supplement 1), S153-S175

Mideplan (n.d.). Índice de desarrollo social. Ministerio de Planificación Nacional y Política Económica. https:// www.mideplan.go.cr/indice-desarrollo-social

Moreno, M., \& Alfaro, E. J. (2018). Valoración socioeconómica del impacto de la variabilidad climática sobre la pesca artesanal en Costa Rica. Uniciencia, 32(1), 18-31. http://dx.doi.org/10.15359/ru.32-1.2

Moreno, M. L., Hidalgo, H. G., \& Alfaro, E. J. (2019) Cambio climático y su efecto sobre los servicios ecosistémicos en dos parques nacionales de Costa Rica, América Central. Revista Iberoamericana de Economía Ecológica, 30(1), 16-38.
Moreno, M., Moya, R., \& Alfaro, E. J. (2017). Actividades socioeconómicas que emplean recursos naturales de la zona marítimo-terrestre y marina en Costa Rica y su relación con la variabilidad climática. Revista de Política Económica para el Desarrollo Sostenible, 2(2), 1-23. http://dx.doi.org/10.15359/peds.2-2.1

Nissan, H., Goddard, L., de Perez, E. C., \& Furlow, J. (2019). On the use and misuse of climate change projections in international development. WIREs Climatic Change 10, e579. https://doi.org/10.1002/wcc.579

Office for the Coordination of Humanitarian Affairs (2020). Costa Rica Population Counts. The Humanitarian Data Exchange. Recuperado de https://data.humdata.org/ dataset/worldpop-population-counts-for-costa-rica.

Pérez-Briceño, P. M., Alfaro, E. J., Hidalgo, H., \& Jiménez, F. (2016). Distribución espacial de impactos de eventos hidrometeorológicos en América Central. Revista de Climatología, 16, 63-75.

PNUD (2020). Índice de Desarrollo Humano (IDH). PNUD Costa Rica. https://www.cr.undp.org/content/ costarica/es/home/library/indice-de-desarrollo-humano--idh-.html

Quesada-Hernández, L. E., Calvo-Solano, O. D., Hidalgo, H. G., Pérez-Briceño, P. M., \& Alfaro, E. J. (2019). Dynamical delimitation of the Central America Dry Corridor (CADC) using drought indices and aridity values. Progress in Physical Geography, 43(5), $627-$ 642. https://doi.org/10.1177/0309133319860224

Quesada-Román, A., \& Villalobos-Chacón, A. (2020). Flash flood impacts of Hurricane Otto and hydrometeorological risk mapping in Costa Rica. Geografisk Tidsskrift-Danish Journal of Geography, 120(2), 142-155. https://doi.org/10.1080/00167223.2020.18 22195

Quesada-Román, A., Villalobos-Portilla, E. \& CamposDurán, D. (2020). Hydrometeorological disasters in urban areas of Costa Rica, Central America. Environmental Hazards, 1-15. https://doi.org/10.1080/ 17477891.2020.179103410.1080/17477891.2020.17 91034.

Quesada-Román, A., Fallas-López, B., HernándezEspinoza, K., Stoffel, M., \& Ballesteros-Cánovas, J.A. (2019). Relationships between earthquakes, hurricanes, and landslides in Costa Rica. Landslides, 16(8), 1539-1550. https://doi.org/10.1007/ s10346-019-01209-4

Retana, J. A., Alvarado, L., Araya, C., Sanabria, N., Solano, J., Solera, M., \& Alfaro, M. (2012). Caracterización del corredor seco en Costa Rica. Tópicos Meteorológicos y Oceanográficos, 11(1), 18-29. 
Rodríguez, A., Alfaro, E. J., \& Cortés J. (2021) Spatial and temporal dynamics of the hydrology at Salinas Bay, Costa Rica, Eastern Tropical Pacific. Revista de Biología Tropical, 69(Suppl. 2), S105-S126.

Ruíz, S., Pérez-Briceño, P. M., Hidalgo, H. G., \& Alfaro, E. J. (2019). Aspectos climáticos del Parque Nacional Santa Rosa. Periodo de enero de 2011 a enero de 2019. Documento Técnico. CIGEFI-UCR. Recuperado de http://hdl.handle.net/10669/81756

Vargas-Sanabria, D., \& Quesada-Román, A. (2018). Influencia geomorfológica en la vulnerabilidad a incendios forestales en el Área de Conservación Guanacaste, Costa Rica. Revista de Ciencias Ambientales, 52(2), 1-15. https://doi.org/10.15359/rca.52-2.1
Veas-Ayala, N., Quesada-Román, A., Hidalgo, H., \& Alfaro, E. (2018). Humedales del Parque Nacional Chirripó, Costa Rica: características, relaciones geomorfológicas y escenarios de cambio climático. Revista de Biología Tropical, 66(4), 1436-1448. https://doi.org/10.15517/rbt.v66i4.31477

Xie, P., \& Arkin, P. (1997). Global precipitation: a 17-year monthly analysis based on gauge observations, satellite estimates, and numerical model outputs. Bulletin of the American Meteorological Society, 78, 2539-2558. https://doi.org/10.1175/1520-0477(1997)078\%3C25 39:GPAYMA\%3E2.0.CO;2 